\title{
ALTERNATIVAS NO CONTROLE QUÍMICO DO Aedes aegypti E Culex quinquefasciatus: ESTUDO PRELIMINAR SOBRE A EFICÁCIA DE PARASITICIDAS DE USO EXTERNO CONTRA LARVAS DE CULICÍDEOS
}

Willian Marinho Dourado Coelho ${ }^{1}$, Claudimir Couto Junior ${ }^{2}$, Vamilton Alvares Santarém ${ }^{3}$, Wilma Aparecida Starke Buzetti ${ }^{4}$

${ }^{1}$ Universidade Estadual Paulista - UNESP, Faculdade de Engenharia de Ilha Solteira, llha Solteira, SP. ${ }^{2}$ Faculdade de Ciências Agrárias de Andradina - FCAA, Andradina, SP. ${ }^{3}$ Universidade do Oeste Paulista - UNOESTE, Presidente Prudente, SP. ${ }^{4}$ Universidade Estadual Paulista Júlio de Mesquita Filho - UNESP, Presidente Prudente, SP

\section{RESUMO}

Este trabalho tem o objetivo de avaliar a eficácia de produtos parasiticidas no controle de larvas de Aedes aegypti e Culex quinquefasciatus. Os produtos Fluazuron 2,5g, Cipermetrina 150g, Flumetrina 1g, Propoxur $1 \mathrm{~g}$, Deltametrina $25 \mathrm{~g}$, Clorpirifós $30 \mathrm{~g}$, Cipermetrina 15g, Fenthion 15g, Diazinon técnico estabilizado $40 \mathrm{~g}$, Fipronil 2,5\%, Triclorfone 97g, Coumafós 3g, Propoxur 2g, Fentione 15g, Amitraz 12,5\% foram preparados conforme recomendações do fabricante, sendo aplicados em recipientes contendo larvas de $A$. aegypti e $C$. quinquefasciatus em ambiente controlado. A inspeção da viabilidade larval foi verificada durante 24 horas. Estes mesmos produtos foram aplicados nos locais de coleta das larvas, sendo os criadouros dos mosquitos vistoriados diariamente durante 30 dias. Foi observado que $100 \%$ das larvas morreram em até 15 minutos, tanto nos recipientes em laboratório quanto no meio ambiente. Nas áreas onde ocorreu a pulverização não se observou presença de mosquitos adultos ou larvas por um período de até 24 dias, dependendo do produto utilizado, da incidência solar e da precipitação local. Nos locais ao abrigo da chuva e luz solar intensa, a ausência de larvas dos mosquitos ocorreu por 30 dias. Podemos concluir com esses resultados que diferentes produtos utilizados em animais como parasiticidas externos foram eficazes contra larvas de culicideos, constituindo-se numa importante ferramenta para controle desses vetores.

Palavras-chave: controle químico; Dengue; febre amarela; febre Chikungunya; Zika vírus.

\section{ALTERNATIVES IN THE CHEMICAL CONTROL OF Aedes aegypti AND Culex quinquefasciatus: PRELIMINARY STUDY ON THE EFFECTIVENESS OF PARASITICIDES OF EXTERNAL USE AGAINST LARVAE OF CULICIDAE}

\begin{abstract}
This study aims to evaluate the effectiveness of parasiticides products in the control of Aedes aegypti and Culex quinquefasciatus larvae. The products Fluazuron 2,5g, Cipermetrina 150g, Flumetrina $1 \mathrm{~g}$, Propoxur $1 \mathrm{~g}$, Deltametrina $25 \mathrm{~g}$, Clorpirifós $30 \mathrm{~g}$, Cipermetrina 15g, Fenthion 15g, Diazinon técnico estabilizado $40 \mathrm{~g}$, Fipronil 2,5\%, Triclorfone 97g, Coumafós 3g, Propoxur 2g, Fentione 15g, Amitraz 12,5\% were prepared according to manufacturer's recommendations, being applied in containers containing larvae of $A$. aegypti and $C$. quinquefasciatus in a controlled environment. Inspection of the larval viability was assessed 24 hours. These same products have been applied in the collection sites of the larvae, and the breeding of mosquitoes inspected daily for 30 days. Was observed that $100 \%$ of the larvae died within 15 minutes, so the containers in the laboratory and into the environment. In areas where spraying was not observed the presence of mosquito adults or larvae for a period of up to 24 days depending on the product used, the location of sunlight and precipitation. In protected sites of rain and strong sunlight, the absence of mosquito larvae occurred for 30 days. We can conclude from these results that different products used in animals as external parasiticides were effective against culicides larvae, constituting an important tool in the control of these vectors.
\end{abstract}

Keywords: chemical control; Chikungunya fever; Dengue; Zika virus; yellow fever. 


\section{INTRODUÇÃO}

O A. aegypti é um importante parasito antropofílico, que atua como vetor da dengue (TAUIL, 2001), febre amarela (BRAGA, 2007), Zika vírus (VASCONCELOS, 2015) e febre Chikungunya (DONALISIO; FREITAS, 2015), causando epidemias em todos os estados brasileiros, podendo estar relacionado com má formação fetal de seres humanos (BRASIL, 2016a), e ocasionando elevado número de óbitos (BRASIL, 2016b,c). Nos programas de controle desse vetor, a utilização de produtos químicos assume relevada importância, tendo sido amplamente utilizado há décadas, o que resultou numa intensa pressão seletiva, com a sobrevivência de parasitos resistentes aos inseticidas (BRAGA, 2007), particularmente ao temefós (LIMA et al., 2006; DINIZ et al., 2014). Uma vez que o produto larvicida de maior utilização no Brasil já não possui a eficácia desejada, este trabalho apresenta 15 produtos químicos utilizados como endectocidas de pequenos e grandes animais e que, além de serem de baixo custo e de fácil acesso à população, após o preparo, resultam na produção de grandes quantidades de inseticidas altamente letais contra larvas e mosquitos adultos, auxiliando no controle desses parasitos, ainda que na rotulagem de fábrica não contenha a indicação para tal finalidade.

O objetivo desse estudo foi avaliar a eficácia de produtos utilizados como parasiticidas de uso externo no controle de larvas de $A$. aegypti e Culex quinquefasciatus na região noroeste do estado de São Paulo, Brasil.

\section{MATERIAIS E MÉTODOS}

Larvas do mosquito $A$. aegypti e $C$. quinquefasciatus foram coletadas diretamente do meio ambiente, em áreas periurbanas e urbanas do município de Andradina (latitude 20ㅇ 53' $43^{\prime \prime}$, longitude 51 22' 46" W, altitude $405 \mathrm{~m}$ ), estado de São Paulo, Brasil, entre janeiro de 2014 e fevereiro de 2016. As variáveis climáticas foram analisadas diariamente on line junto ao Instituto Nacional de Pesquisas Espaciais (INPE, 2016). As larvas foram identificadas após montagem em lâmina, de acordo com chaves taxonômicas (FORATTINI, 1962). Estas amostras foram separadas em alíquotas com 30 larvas cada, em recipientes contendo água do local de origem (Figura

1).

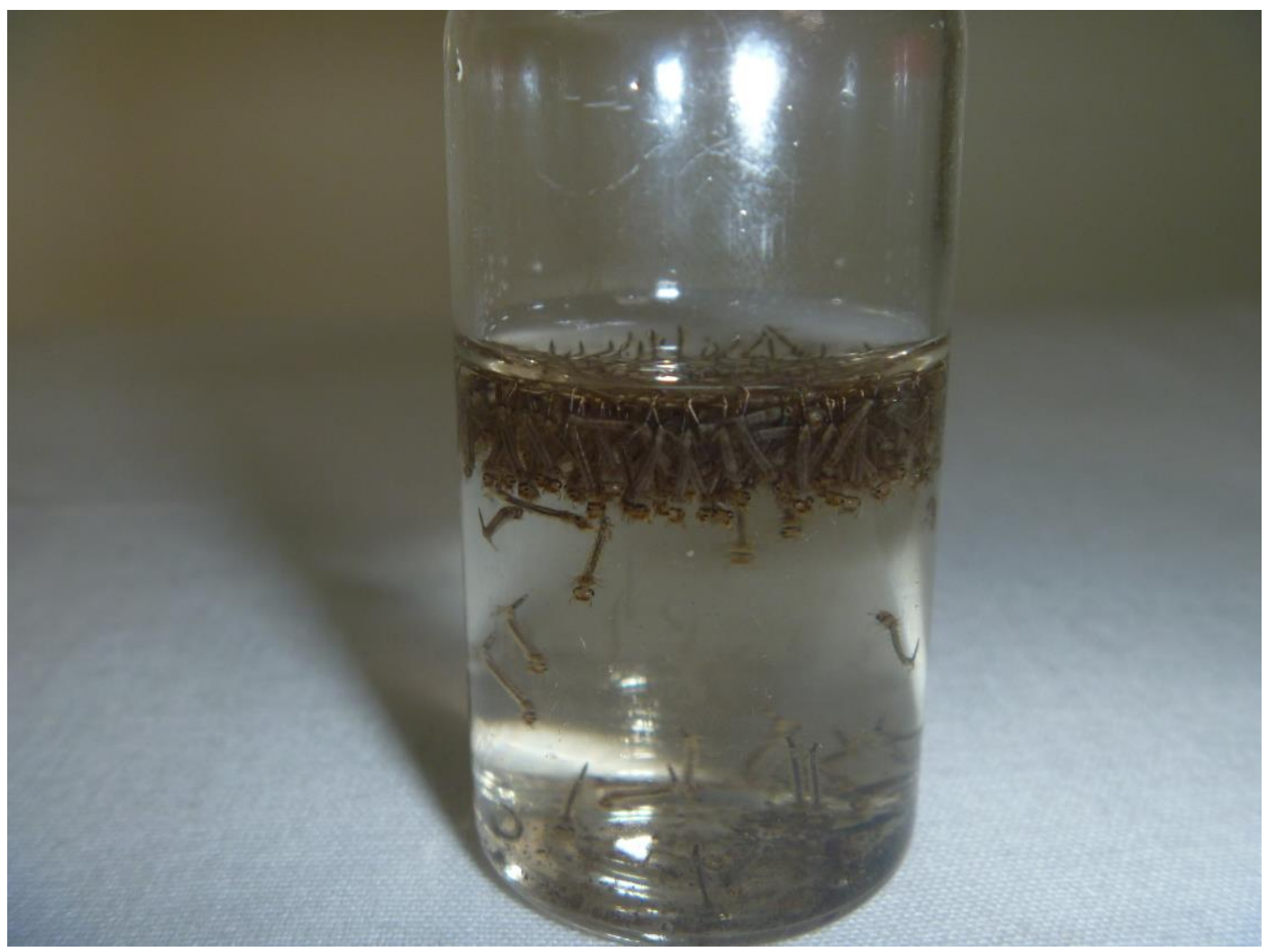

Figura 1. Larvas de culicídeos capturadas em criadouros naturais no perímetro urbano do município de Andradina, São Paulo, 2016. 
As larvas restantes foram deixadas em criadouros para serem submetidas à aplicação dos produtos químicos in loco. Os grupos tratados, com 30 larvas cada, foram colocados em potes coletores universais com $50 \mathrm{~mL}$ 's de água, sendo submetidas a aplicação isolada dos produtos Fluazuron 2,5g, Cipermetrina 150g, Flumetrina 1g, Propoxur 1g, Deltametrina 25g,
Clorpirifós 30g, Cipermetrina 15g, Fenthion 15g, Diazinon técnico estabilizado $40 \mathrm{~g}$, Fipronil 2,5\%, Triclorfone $97 \mathrm{~g}$, Coumafós $3 \mathrm{~g}$, Propoxur $2 \mathrm{~g}$, Fentione $15 \mathrm{~g}$, Amitraz 12,5\%. O grupo controle, com igual número de larvas, recebeu apenas água mineral de bebida (Figura 2). Foram realizadas cinco repetições para cada tratamento.

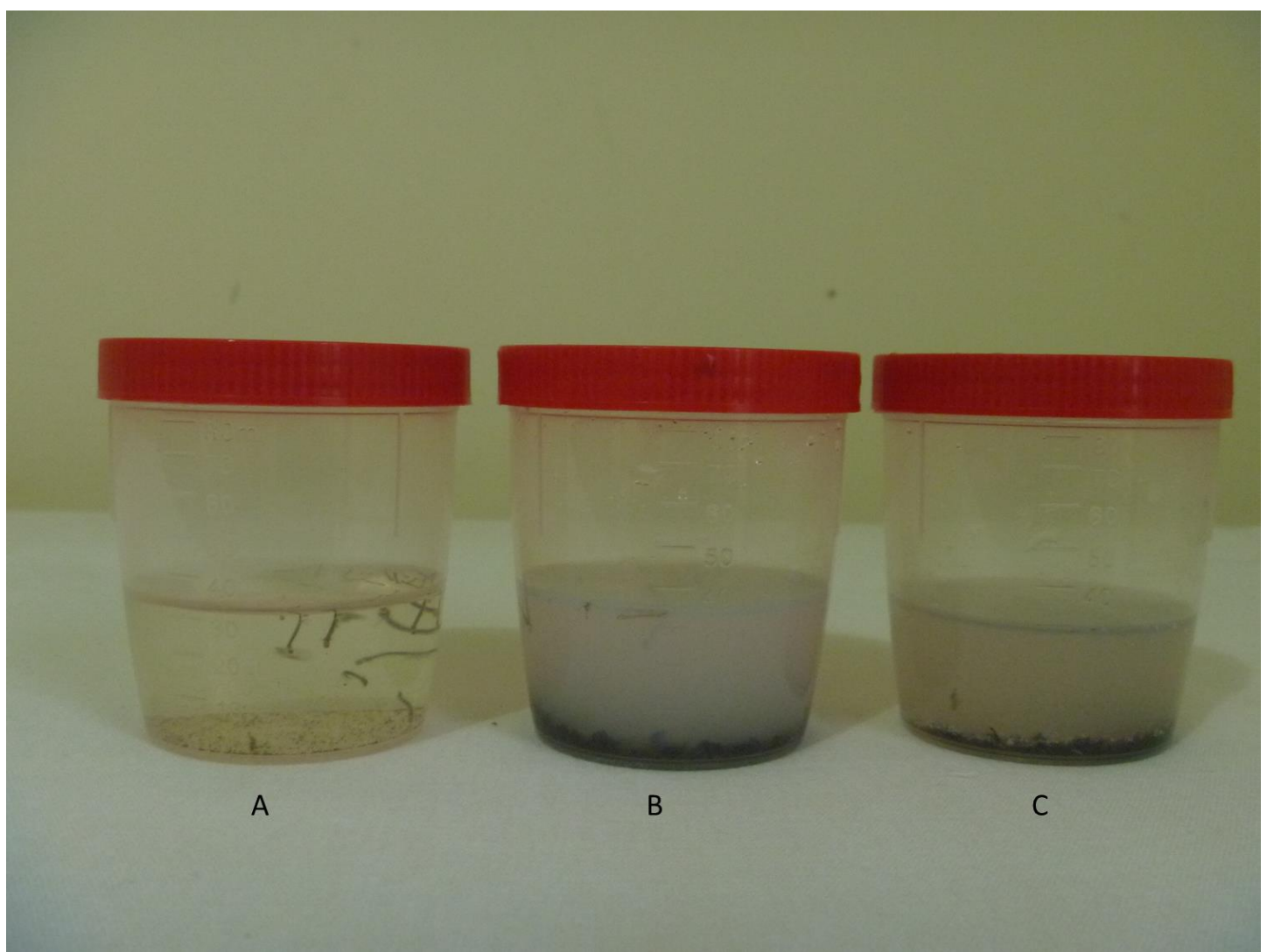

Figura 2. Esquema de aplicação de parasiticidas externos em larvas de culicídeos. Grupo controle (A), grupo Amitraz 12,5\% (B), Grupo Diazinon técnico estabilizado 40g (C), 2016.

Antes da aplicação, os produtos foram previamente preparados (diluídos) conforme recomendações dos fabricantes, e aplicados com a utilização de uma pipeta Pasteur (20 gotas $1 \mathrm{~mL}$ ). No meio ambiente (área urbana e periurbana), em domicílios e quintais, a pulverização foi efetuada com a utilização de pulverizador costal manual de $10 \mathrm{~L}\left(\right.$ Guarany $^{\circledR}$ ) em torno e dentro dos recipientes que atuam como criadouros. Os produtos com apresentação em pó foram pesados e polvilhados sobre as amostras previamente aliquotadas (1 colher das de sopa rasa - 15g) e também nos criadouros (50g por $\mathrm{m}^{2}$ ).

A letalidade dos produtos foi considerada após a constatação da morte de $100 \%$ das larvas, observando-se sua plena imobilização no fundo dos recipientes, sendo classificada como de eficácia nula (---) na ausência da morte; ruim (+) com morte em até 24 horas; regular (++) com morte em até 12 horas; bom (+++) com morte em até uma hora e excelente $(++++)$ com morte instantânea ou inferior a 60 minutos.

$O$ efeito residual e repelente foi avaliado por meio de inspeção visual periódica dos criadouros durante 30 dias ininterruptos, sendo classificado como elevada (na ausência de larvas por até 30 dias), moderada (ausência de larvas por até 15 dias), baixa (ausência de larvas por até cinco dias), nula (quando foram observadas larvas em qualquer um dos quatro primeiros dias póspulverização).

A análise estatística entre a eficácia e a repelência dos produtos contra as larvas foi analisada por meio do teste Qui-quadrado, com nível de significância de $5 \%$. 


\section{RESULTADOS}

Foi observado que $100 \%$ das larvas morreram em até cinco minutos (Tabela 1), tanto nos recipientes mantidos em laboratório quanto no ambiente. Nas áreas onde ocorreu a pulverização, não se observou presença de mosquitos adultos ou larvas por um período de até 24 dias, dependendo do produto utilizado, da incidência solar e da precipitação local. Nos locais ao abrigo da chuva e luz solar intensa, não se observou novos focos por um período de 30 dias, quando se deu a conclusão do presente estudo. Não foi verificada diferença estatística entre os produtos utilizados e a letalidade ( $p \leq 0,05 \%)$. Houve diferença estatística significante entre a repelência dos demais produtos em relação ao Amitraz

$12,5 \%$.

Tabela 1. Princípios ativos utilizados contra larvas de Aedes aegypti e Culex quinquefasciatus na cidade de Andradina, São Paulo, Brasil, 2016.

\begin{tabular}{l|c|c}
\hline Produto/princípio ativo & Letalidade & Repelência $^{\text {a }}$ \\
\hline Água de bebida (controle) & --- & Nula \\
Fluazuron 2,5g & ++++ & Moderada \\
Cipermetrina 150g/L & ++++ & Moderada \\
Flumetrina 1g & ++++ & Moderada \\
Propoxur 1g* & ++++ & Moderada \\
Deltametrina 25,0 g g/L & ++++ & Moderada \\
Fenthion 15,0g, Cipermetrina 15,0g, Clorpirifós 30,0g & ++++ & Moderada \\
Diazinon técnico estabilizado 40g* & ++++ & Moderada \\
Fipronil 2,5\% & ++++ & Moderada \\
Triclorfone 97,0g* & ++++ & Moderada \\
Coumafós 3g, Propoxur 2g* & ++++ & Elevada \\
Fentione 15g & ++++ & Moderada \\
Amitraz 12,5\% & ++++ & Baixa \\
\hline
\end{tabular}

Legenda: Letalidade: ---- eficácia nula; + ruim; ++ regular; +++ bom; ++++ excelente.

Repelência: elevada (na ausência de larvas por até 30 dias), moderada (ausência de larvas por até 15 dias), baixa (ausência de larvas por até cinco dias), nula (quando foram observadas larvas em qualquer um dos quatro primeiros dias pós-pulverização).

${ }^{a}$ Diferença estatística significativa $p \leq 0,05 \%$.

Apresentação em pó ( $\left.{ }^{*}\right)$

\section{DISCUSSÃO}

Nossos resultados sugerem que diferentes marcas de produtos químicos à base de organofosforados e piretróides disponíveis comercialmente como parasiticidas e uso externo possuem elevada eficácia no controle de larvas desse parasito, com ação larvicida, adulticida, ovicida e repelente nos locais de foco desse vetor.

As doenças transmitidas pelo $A$. aegypti causam grande impacto em saúde pública, com sérias epidemias em todo território nacional e em grande parte do mundo (BRASIL, 2001). Desse modo, a aplicação de inseticidas tem sido empregada há décadas no combate à esse vetor, especialmente organofosforados como o temefós (BRASIL, 2001; CARVALHO et al., 2004), malation (COLEONE, 2014), piretóides e carbamatos (BRAGA; VALLE, 2007).

Lima et al. (2006) comprovaram a alta resistência das larvas de $A$. aegypti ao temefós em diversas localidades de Fortaleza (LIMA et al., 2006), sendo constatado que, em mais da metade dos bairros aonde foram feitas as coletas de amostras de larvas, a mortalidade dos insetos expostos ao produto foi nula. Diniz et al. (2014) também encontraram altos percentuais de resistência ao temefós em diversos bairros de Campina Grande, Paraíba.

Desta forma, em razão dos numerosos surto de doenças transmitidas pelo mosquito $A$. aegypti na atualidade, em detrimento das numerosas medidas de prevenção e controle adotadas pela comunidade e pelos órgãos públicos, além da ocorrência de resistência desse artrópode aos inseticidas existentes, a apresentação de novos produtos com elevada eficácia contra as formas evolutivas desse parasito, possibilitam implementar novos planos de combate ao vetor, resultando na interrupção de seu ciclo biológico por inviabilizar a eclosão dos ovos, ocasionando a morte das larvas e 
inativando e repelindo os insetos adultos dos locais com água estagnada.

\section{REFERÊNCIAS}

BRAGA, I.A.; VALLE, D. Aedes aegypti: inseticidas, mecanismo de ação e resistência. Epidemiol Serv Saúde, v.16, n.4, p.279-93, 2007. DOI: http://dx.doi.org/10.5123/S167949742007000400006.

BRASIL. Fundação Nacional de Saúde. Dengue. Instruções para pessoal de combate ao vetor. Brasília: 2001. (Manual de normas técnicas)

BRASIL. Ministério da Saúde. Secretaria de Vigilância em Saúde. Boletim Epidemiológico. Monitoramento dos casos de microcefalias no Brasil até a semana epidemiológica. n.51, 2016a. Disponível em: <http://portalsaude.saude.gov.br/images/pdf/20 16/janeiro/11/2015-053-para-substituir-na-p-gina.pdf>

BRASIL. Ministério da Saúde. Secretaria de Vigilância em Saúde. Boletim Epidemiológico. Monitoramento dos casos de dengue, febre de chikungunya e febre pelo vírus Zika até a Semana Epidemiológica. 51, 2016b. Disponível em:

$<$ http://portalsaude.saude.gov.br/images/pdf/20 16/janeiro/15/svs2016-be002-dengue-se51.pdf>

BRASIL. Ministério da Saúde. Secretaria de Vigilância em Saúde. Boletim Epidemiológico. Monitoramento dos casos de dengue, febre de chikungunya e febre pelo vírus Zika até a Semana Epidemiológica. 52, 2016c. Disponível em:

<http://portalsaude.saude.gov.br/images/pdf/20 16/janeiro/15/svs2016-be003-dengue-se52.pdf>

CARVALHO, M.S.L.; CALDAS, E.D.; DEGALLIER, N.; VILARINHOS, P.T.R.; SOUZA, L.C.K.R.; YOSHZAWA, M.A.C.; KNOX, M.B.; OLIVEIRA, C. Susceptibility of Aedes aegypti larvae to the insecticide temephos in the Federal District, Brazil. Rev Saúde Pública, v.38, n.5, p.1-6, $2004 . \quad$ DOI: https://dx.doi.org/10.1590/S0034-

$\underline{89102004000500002}$

\section{CONCLUSÃO}

Conclui-se que diferentes produtos utilizados em animais para promover o controle de ectoparasitos e endoparasitos foram eficazes contra larvas de $A$. aegypti, constituindo-se numa potencial ferramenta para controle desse vetor.

COLEONE, A,C. Avaliação da dissipação do inseticida malation utilizado em nebulização a ultrabaixo volume no controle da dengue: avaliação ecotoxicológica e de risco ambiental. 2014. Dissertação (Mestrado) - Universidade de São Paulo. Faculdade de Saúde Pública, 2014. https://doi.org/10.11606/D.6.2014.tde05062014-134246

DINIZ, M.M.C.S.L.; HENRIQUES, A.D.S.; LEANDRO, R.S.; AGUIAR, D.L.; BESERRA, E.B. Resistência de Aedes aegypti ao temefós e desvantagens adaptativas. Rev Saúde Pública, v.48; n.5, p.77582, 2014. https://doi.org/10.1590/50034$\underline{8910.2014048004649}$

DONALISIO, M.R.; FREITAS, A.R.R. Chikungunya no Brasil: um desafio emergente. Rev Bras Epidemiol, v.18, n.1, p.283-85, 2015. DOI: https://dx.doi.org/10.1590/1980-

5497201500010022

FORATTINI, O.P. Entomologia médica. São Paulo: Faculdade de Higiene e Saúde Pública, 1962. v.l.

INPE - Instituto Nacional de Pesquisas Espaciais. Centro e Previsão de Tempo e Estudos Climáticos. 2016. <http://www.cptec.inpe.br/cidades/tempo/474>.

LIMA, E.P.; FILHO, A.M.O.; LIMA, J.W.O.; JÚNIOR, A.N.R.; CAVALCANTI, L.P.G.; PONTES, R.J.S. Resistência do Aedes aegypti ao temefós em municípios do estado do Ceará. Rev Soc Bras Med Trop., v.39, n.3, p.259-63, 2006. DOI: https://dx.doi.org/10.1590/S003786822006000300006

TAUIL, P.L. Urbanização e ecologia do dengue. Cad Saúde Pública, v.17, p.99-102, 2001. https://doi.org/10.1590/S0102$\underline{311 \times 2001000700018}$

VASCONCELOS, P.F.C. Doença pelo vírus Zika: um novo problema emergente nas Américas? Rev Pan-Amazônica de Saúde, v.6, n.2, p.9-10, 2015. Disponível 
<http://scielo.iec.pa.gov.br/scielo.php?script=sci _arttext\&pid=S2176-

62232015000200001\&lng=pt\&nrm=iso>
Recebido para publicação em 16/12/2016 Revisado em 18/02/2017

Aceito em 27/03/2017 